American Journal of Applied Sciences 3 (10): 2037-2041, 2006

ISSN 1546-9239

(c) 2006 Science Publications

\title{
Chaotic Attitude Tumbling of Satellite in Magnetic Field
}

\author{
Rashmi Bhardwaj and Parsan Kaur \\ Department of Mathematics, School of Basic and Applied Sciences \\ Guru Gobind Singh Indraprastha University, Kashmere Gate, Delhi-110006, India
}

\begin{abstract}
In this study, the half width of the chaotic separatrix has been estimated by chrikov's criterion. Through surface of section method, it has been observed that the magnetic torque parameter, the eccentricity of the orbit and the mass distribution parameter play an important in changing the regular motion into chaotic one.
\end{abstract}

Key words: Chaos, celestial mechanics, solar system, periodic orbits, poincare section

\section{INRODUCTION}

Bhardwaj $^{[1]}$ has discussed chaos in non-linear planar oscillation of a satellite under the influence of third-body torque. Sidlichovsky ${ }^{[2]}$ has discussed the existence of a chaotic region which is formed by trajectories crossing a critical curve which corresponds to the separatrix of fast pendulum motion. Tiscareno ${ }^{[3]}$ carried out extensive numerical orbit integrations to probe the long-term chaotic dynamics of the 2:3 (Plutinos) and 1:2 (Twotinos) mean motion resonances with Neptune. Kauprianov and Shevchenko ${ }^{[4]}$ studied the problem of observability of chaotic regimes in the rotation of planetary satellites. Contopoulos and Efstathiou $^{[5]}$ studied Escapes and Recurrence in a Simple Hamiltonian System. They studied a simple dynamical system with escapes using a suitably selected surface of section. Selaru et al. ${ }^{[6]}$ studied Chaos in Hill's generalized problem from the solar system to black holes. Carruba et al. ${ }^{[7]}$ have discussed Chaos and Effects of Planetary Migration for the Saturnian Satellite Kiviuq.

Equation of motion: The equation of motion for the non-linear motion of a satellite under the influence of magnetic torque in an elliptic orbit as obtained as

$$
\frac{d^{2} \theta}{d t^{2}}+\frac{\mu}{2 r^{3}} n^{2} \sin \delta-\frac{\varepsilon \mu}{2 r^{3}} \sin 2\left(\Omega-\alpha_{m}+v\right)=0
$$

which can also be written as

$$
\begin{aligned}
& (1+e \cos v) \frac{d^{2} q}{d v^{2}}-2 e \sin v \frac{d q}{d v} \\
& -4 e \sin v+n^{2} \sin q=\varepsilon \sin \left(a_{1}+b v\right)
\end{aligned}
$$

Estimation of resonance width: As $\mathrm{r}$ and $\mathrm{v}$ are periodic in time and as $\theta=v+\frac{\delta}{2}$, using Fourier like
Poisson-Series as discussed in Bhardwaj and Tuli ${ }^{[1]}$, Equation (1), becomes

$$
\begin{gathered}
\frac{d^{2} \theta}{d t^{2}}+\frac{w_{0}^{2}}{2} \sum H\left(\frac{m}{2}, e\right) \sin (2 \theta-m t)-\frac{\varepsilon}{2} \\
\sum H\left(\frac{m}{b}, e\right) \sin \left(2\left(\Omega_{0}-\alpha_{1}\right)+\frac{m t}{b_{1}}\right)=0
\end{gathered}
$$

The half integers $\frac{m}{2}$ will be denoted by $p$. Resonances occur whenever one of the arguments of the sine or cosine functions is nearly stationary i.e., whenever $\left|\frac{d \theta}{d t}-p\right|<<\frac{1}{2}$. Using slowly varying resonance variable, $v_{p}=\theta-p t$, holding $v_{p}$ fixed and averaging for small $w_{0}$, then, Equation (2) becomes

$$
\begin{aligned}
& \frac{d^{2} v_{p}}{d t^{2}}+\frac{w_{0}^{2}}{2} H(p, e) \sin 2 v_{p} \\
& -\frac{\varepsilon}{2} H\left(\frac{p}{b}, e\right) \sin 2\left(\Omega_{0}-\alpha_{1}+\frac{2 p t}{b}\right)=0
\end{aligned}
$$

which is a equation of perturbed pendulum perturbed by a force $\frac{\varepsilon}{2} H\left(\frac{p}{b}, e\right) \sin 2\left(\Omega_{0}-\alpha_{1}+\frac{2 p t}{b}\right)$

When $\varepsilon \neq 0$ the Equation.(3) becomes

$$
\frac{d^{2} x_{p}}{d t^{2}}+f^{\prime}\left(x_{p}\right)=m_{p} \phi^{\prime}(t, c)
$$

Where

$$
\begin{aligned}
& f^{\prime}\left(x_{p}\right)=k_{1 p}{ }^{2} \sin x_{p}, m_{p}=k_{2 p}{ }^{2}, \phi^{\prime}(t, c)=\sin \frac{4 p}{b}\left(t+a_{3}\right) \\
& x_{p}=2 v_{p}, \\
& k_{1 p}{ }^{2}=w_{0}{ }^{2} H(p, e), k_{2 p}{ }^{2}=\varepsilon H\left(\frac{p}{b}, e\right), a_{3}=\frac{\left(\Omega_{0}-\alpha_{1}\right) b}{2 p}
\end{aligned}
$$

Corresponding Author:

Dr. Rashmi Bhardwaj, Department of Mathematics, School of Basic and Applied Sciences, Guru Gobind Singh Indraprastha University, Kashmere Gate, Delhi - 110006, India 
Am. J. Appl. Sci., 3 (10): 2037-2041, 2006

For the unperturbed part of Equation (4), $\left(\frac{d x_{p}}{d t}\right)^{2}=c_{1 p}+2 k_{1 p}{ }^{2} \cos x_{p}$, where $c_{1 p}$ is constant of integration. The motion to be real if $c_{1 p}+2 k_{1 p}{ }^{2} \geq 0$. There are three Categories of motion depending upon $c_{1 p}>2 k_{1 p}{ }^{2}, c_{1 p}<2 k_{1 p}{ }^{2}$ and $c_{1 p}=2 k_{1 p}{ }^{2}$

Category-I $c_{1 p}>2 k_{1 p}{ }^{2}$

If $\frac{d x_{p}}{d t} \neq 0$, the motion is said to be revolution and unperturbed solution is

$x_{p}=l_{p}+c_{1 p} \sin l_{p}+O\left(c_{1 p}{ }^{2}\right)$,

where, $\quad l_{p}=n_{p} t+\varepsilon_{1}, \quad c_{1 p}=\frac{k_{1 p}{ }^{2}}{n_{p}^{2}}$ and

$\frac{1}{n_{p}}=\frac{1}{2 \pi} \int_{0}^{2 \pi} \frac{d x_{p}}{\left(c_{1 p}+2 k_{1 p}{ }^{2} \cos x_{p}\right)^{1 / 2}}$,

$c_{1 p}, \varepsilon_{1}$ are arbitrary constants and $l_{p}$ is an argument. Using theory of variation of parameters, since $m_{p}$ and $k_{1 p}{ }^{2}$ are small quantities, so rejecting second or higher order terms $\frac{d c_{1 p}}{d t} \cong 0 \Rightarrow c_{1 p}$ is a constant upto second order of approximation.

and $\frac{d^{2} l_{p}}{d t^{2}} \cong m_{p} \sin \frac{4 p}{b}\left(t+a_{3}\right) \cong 2 m_{p} \sin \frac{2 p l_{p}}{b n_{p}}$. If we take $\frac{2 p l_{p}}{b n_{p 0}}=x_{p}$, then

$\left(\frac{d x_{p}}{d t}\right)^{2}=c_{2 p}+2 k_{3 p}{ }^{2} \cos x_{p}$, where $c_{2 p}$ is constant of integration and $k_{3 p}{ }^{2}=\frac{4 \varepsilon p}{b n_{p 0}}$.

Again, we get three types of motion, Type I, II is that in which $\frac{d x_{p}}{d t}>0,<0$, Type III is that in which $\frac{d x_{p}}{d t}=0$, at 0 or $\pi$,

For type-I, our solution is

$x_{p}=N_{p} t+\varepsilon_{2}+\frac{k_{3 p}{ }^{2}}{N_{p}{ }^{2}} \sin \left(N_{p} t+\varepsilon_{2}\right)$

$+\frac{k_{3 p}^{4}}{8 N_{p}^{4}} \sin 2\left(N_{p} t+\varepsilon_{2}\right)+\ldots \ldots \ldots$

where $\frac{1}{N_{p}}=\frac{1}{2 \pi} \int_{0}^{2 \pi} \frac{d x_{p}}{\left(c_{2 p}+2 k_{3 p}{ }^{2} \cos x_{p}\right)^{1 / 2}}$, which is the case of revolution.
For the type II, the solution is $x_{p}=\lambda \sin \left(p^{\prime} t+\lambda_{0}\right)$ where $p^{\prime}=2 \sqrt{\frac{\varepsilon p}{b n_{p 0}}}$.

$\lambda$ and $\lambda_{0}$ being arbitrary constants. This is the case of liberation.

Type III occurs when $c_{2 p}=2 k_{3 p}{ }^{2}=\frac{8 \varepsilon p}{b n_{0}}$

The solution is $x_{p}+\pi=4 \tan ^{-1} e^{k_{3} t}+\alpha_{0}$, where $\alpha_{0}$ is an arbitrary constant and the other having a particular value. When $t \rightarrow \pm \infty, x_{p} \rightarrow \pm \pi$, at both places, $\left(\frac{d x_{p}}{d t}\right)=0$ and all higher derivatives of $x_{p}$ approach to zero. This is the case of infinite period separatrix which is asymptotic forward and backward in time to the unstable equilibrium.

Category II: $c_{1 p}<2 k_{1 p}{ }^{2}$

In this case unperturbed solution is

$x_{p}=c_{1 p} \sin l_{p}+\frac{c_{1 p}{ }^{3}}{192} \sin 3 l_{p}+\ldots \ldots \ldots \ldots$.

where,

$l_{p}=n_{p} t+\varepsilon_{1}$,

$n_{p}=k_{1 p}\left[1-\frac{1}{16} c_{1 p}{ }^{2}+\ldots \ldots \ldots.\right], c_{1 p}$ and $\varepsilon_{1}$ are arbitrary constants.

In case of perturbed equation, we get

$k=n_{p} c_{1 p} \Rightarrow k \cong k_{1 p} c_{1 p}$

and

$\frac{d c_{1 p}}{d t} \cong \frac{m_{p}}{k_{1 p}} \cos l_{p} \sin \frac{4 p}{b}\left(t+a_{3}\right)$

Now, $\frac{d l_{p}}{d t} \cong k_{1 p}-\frac{m_{p}}{k_{1 p} c_{1 p}} \sin l_{p} \sin \frac{4 p}{b}\left(t+a_{3}\right)$

$\frac{d^{2} l_{p}}{d t^{2}} \cong-\frac{4 p m_{p}}{b k_{1 p} c_{1 p}} \sin l_{p} \cos \frac{4 p}{b}\left(\frac{l_{p}-\varepsilon_{1}}{n_{p}}+a_{3}\right)$

In the first approximation of $n_{p}=n_{p 0}, c_{1 p}=c_{1 p 0}$, we get

$\frac{d^{2} l_{p}}{d t^{2}} \cong-\frac{4 p m_{p}}{b k_{1 p} c_{1 p 0}} \sin l_{p} \cos \frac{4 p}{b}\left(\frac{l_{p}-\varepsilon_{1}}{n_{p 0}}+a_{3}\right)$

As a special case, let us assume that $\frac{2 p}{b}\left(\frac{l_{p}-\varepsilon_{1}}{n_{p 0}}+a_{3}\right)=\frac{n_{1} \pi}{2}, n_{1} \in I$.

When $n_{1}$ is odd, then, $\frac{d^{2} l_{p}}{d t^{2}}+k_{4 p}{ }^{2} \sin l_{p}=0, \quad k_{4 p}{ }^{2}=-\frac{4 p m_{p}}{b k_{1 p} c_{1 p 0}}>0$ as $m<0$ 

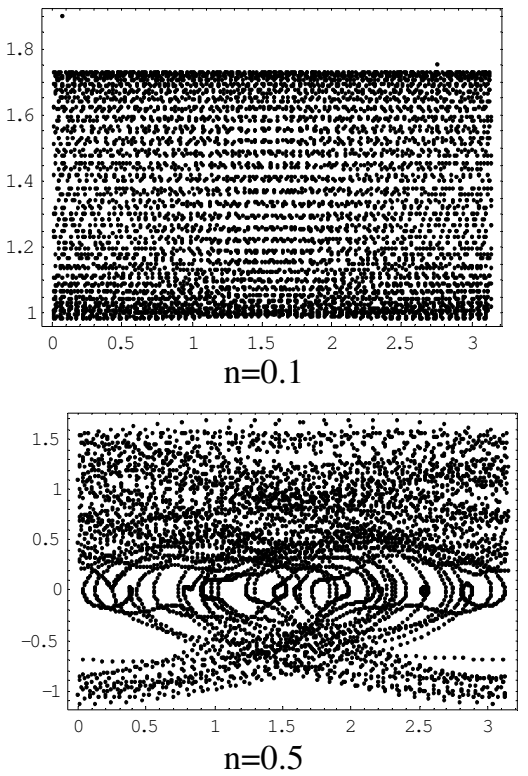
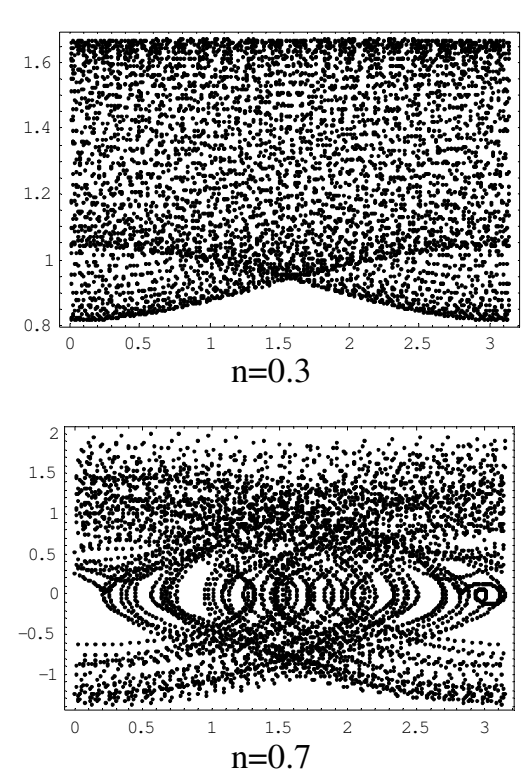
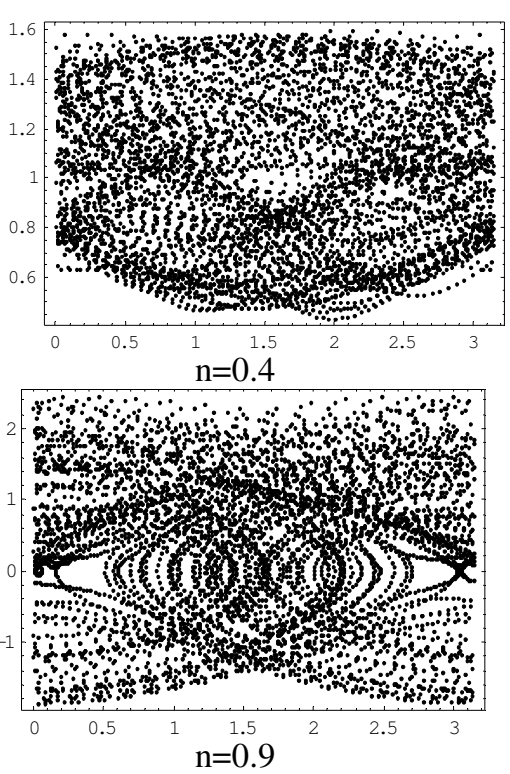

Fig. 1: $\quad$ Surface of sections for $\mathrm{e}=0.0549, \varepsilon=0.001, a_{1}=0.1153$ at $\mathrm{n}=0.1,0.3,0.4,0.5,0.7,0.9$
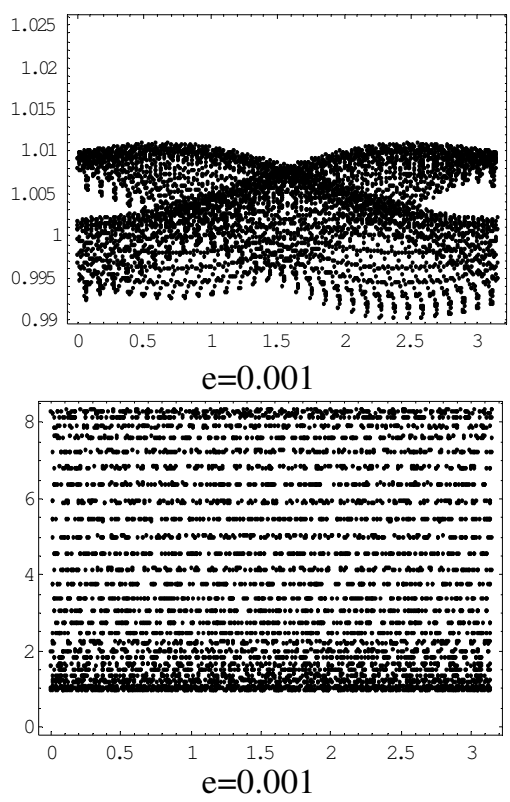
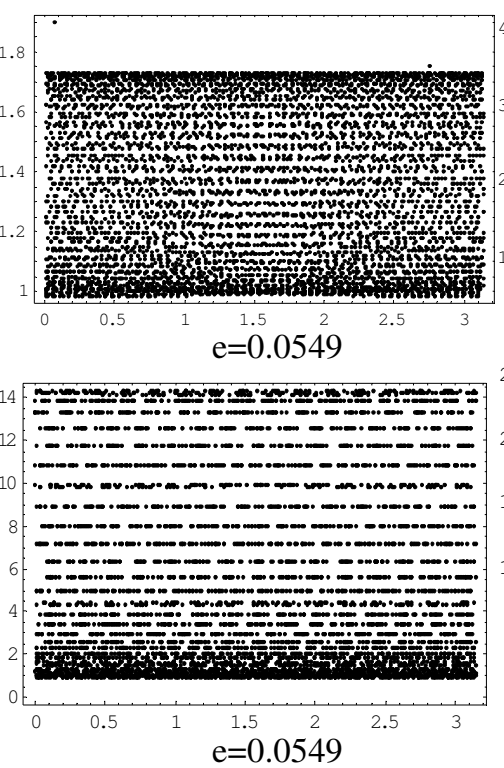
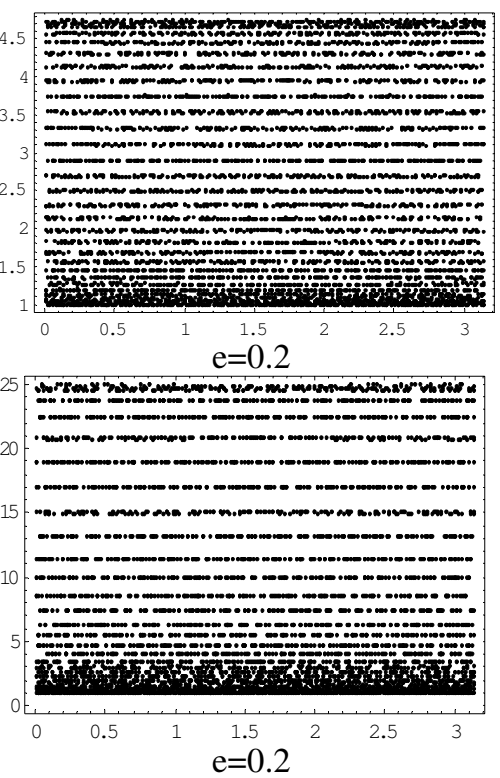

Fig. 2: $\quad$ Surface of sections for $\mathrm{n}=0.1, \varepsilon=0.001, a_{1}=0.1153$ at $\mathrm{e}=0.001,0.0549,0.2,0.3,0.4,0.5$

which is again the equation of pendulum. As in previous case this equation gives us revolution, liberation and infinite period separatrix motion.

On the other hand, if $n_{1}$ is even, then, $\frac{d^{2} l_{p}}{d t^{2}}=-\frac{4 p m_{p}}{b k_{1 p} c_{1 p 0}} \sin l_{p}=k_{4 p}{ }^{2} \sin l_{p}$

When $l_{p}$ is small, the solution of above equation is given by $l_{p}=e^{k_{4} t}+e^{-k_{4} t}$.

Category III: $c_{1 p}=2 k_{1 p}{ }^{2}$
The unperturbed solution is $x_{p}+\pi=4 \tan ^{-1} e^{k_{1 p} t}+\alpha_{0}$, where $\alpha_{0}$ is an arbitrary constant and the other having a specific value. This is the case of infinite period separatrix which is asymptotic forward and backward in time to the unstable equilibrium.

Near the infinite period separatrix broadened by the high frequency term into narrow chaotic band ${ }^{[9]}$, for small $\mathrm{n}$, the half width of the chaotic separatrix is given by $\omega_{1}=\frac{I_{1}-I_{1}^{s}}{I_{1}^{s}}=4 \pi \varepsilon \frac{1}{n^{3}} e^{-(\pi / 2 n)}$. Here, $\omega_{1}$ increases both with $\varepsilon$ and $n$. An estimate of $n$ at which the wide 

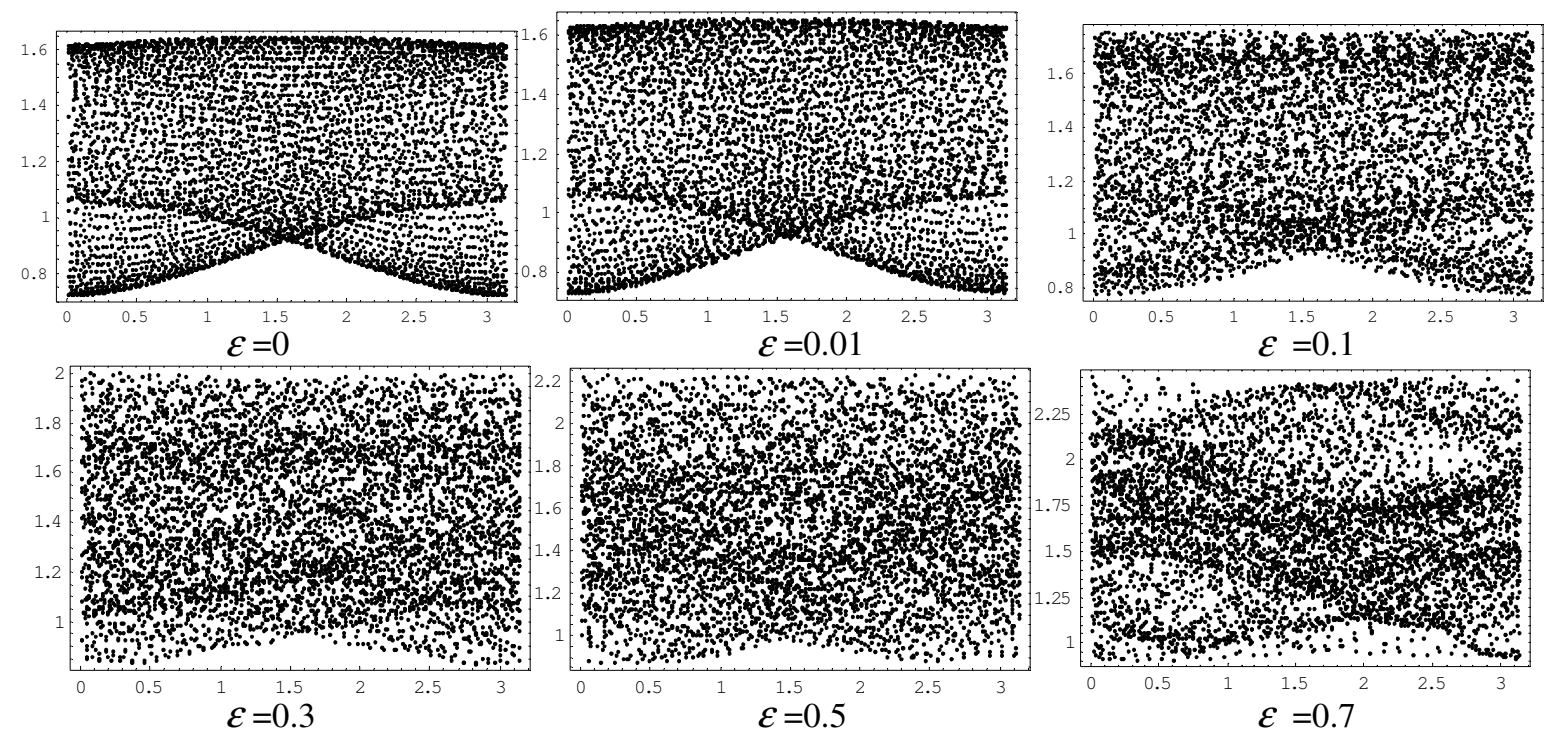

Fig. 3: Surface of sections for $\mathrm{n}=0.347$, $\mathrm{e}=0.0549, a_{1}=0.1153$ at $\varepsilon=0,0.01,0.1,0.3,0.5,0.7$

spread chaotic behaviour can be observed is given by using the Chrikov's overlap criterion. This criterion states that when the sum of two unperturbed half-widths equals the separation of resonance centers, large-scale chaos ensues. In the spin-orbit problem the two resonances with the largest widths are the $p=1$ and $p=$ $3 / 2$ states. For these two states the resonance overlap criterion becomes

$$
\begin{aligned}
& n^{R O} \sqrt{|H(1, e)|}+n^{R O} \sqrt{|H(3 / 2, e)|}=\frac{1}{2} \text { or } \\
& n^{R O}=\frac{1}{2+\sqrt{14 e}} .
\end{aligned}
$$

For $e=0.0549$ the mean eccentricity of Artificial satellite, the critical value of $n$ above which large-scale chaotic behaviour is expected is $n^{R O}=0.347$.

The spin orbit phase space: Using Poincare surface of section by looking at the trajectories stroboscopically with period $2 \pi$. The section has been drawn with versus $v$ at every periapse passage. Since the orientation denoted by $\theta$ is equivalent to the orientation denoted by $\pi+\theta$, we have, therefore, restricted the interval from 0 to $\pi$. In Fig. 1-3, we have plotted $\frac{d \theta}{d v}$ versus $\theta$, at every periapse passage. It may be observed that the chaotic separatrix surrounds each of the resonance states and each of these chaotic zones is separated from others by non-resonant quasi-periodic rotation trajectories. From Fig. 1-3, it is observed that as n, e, $\mathcal{E}$ increases, the regular curves disintegrate respectively and this disintegration increases with the increase in $\mathrm{n}$, $\mathrm{e}, \boldsymbol{\varepsilon}$.

\section{CONCLUSION}

It is also observed that the magnetic torque plays a very significant role in changing the motion of revolution into liberation or infinite period separatrix. The half width of the chaotic sepratrices estimated by Chirikov's criterion is not affected by the magnetic torque. It is further observed that in the spin-orbit phase the regular curves start disintegrating due to magnetic torque, the increase in the eccentricity and the irregular mass distribution of the satellite and this disintegration increases with the increase in $\varepsilon, \mathrm{n}$ and e. It has been observed that Artificial satellite's spin orbit phase space is dominated by a chaotic zone which increases further due to magnetic torque.

\section{REFERENCES}

1. Bhardwaj, R. and R. Tuli, 2005. Non-Linear Planar Oscillation of a Satellite Leading to Chaos Under the Influence of Third-body Torque. Mathematical Models and Methods for Real World Systems. Eds., Furati, Nashed, Siddiqi, Chapman \& Hall/ CRC Publication, pp: 301-336.

2. Sidlichovský, M., 2005. A non-planar circular model for the 4/7 resonance. Celestial Mechanics and Dynamical Astronomy, 93: 167-185.

3. Tiscareno, M.S., 2005. Chaotic diffusion in the outer solar system and other topics. Ph. D. Thesis, pp: 151. United States-Arizona, The University of Arizona. Publication Number: AAT 3145138. DAI-B 65/09, pp: 4623. 
4. Kauprianov, V.V. and I.I. Shevchenko, 2005. Rotational dynamics of planetary satellites: A survey of regular and chaotic behavior. Icarus, 176: 224-234.

5. Contopoulos, G. and K. Efstathiou, 2004. "Escapes and recurrence in a simple Hamiltonian system. Celestial Mechanics, 88: 163-183.

6. Selaru, D., V. Mioc, C. Cucu-Dumitrescu and M. Ghenescu, 2005. Chaos in Hill's generalized problem: from the solar system to black holes. Astronomische Nachrichten, 326: 356-361.
7. Carruba, V., D. Nesvorný, J.A. Burns, M. Cuk and K. Tsiganis, 2004. Chaos and the effects of planetary migration on the orbit of s/2000 S5 Kiviuq. The Astronomical J., 128: 1899-1915.

8. Bhardwaj, R. and P. Kaur, 2006. Satellite's motion under the effect of magnetic torque. Am. J. Appl. Sci., (In press).

9. Chirikov, B.V., 1969. Research concerning the theory of non-linear resonance and stochasticity. Nuclear Physics section of the Siberian Academy of Sciences, Report 267. (In Russian). 